\title{
Color Image Segmentation Using a Semi-wrapped Gaussian Mixture Model
}

\author{
Anandarup Roy ${ }^{1}$, Swapan K. Parui ${ }^{1}$, Debyani Nandi ${ }^{2}$, and Utpal Roy ${ }^{3}$ \\ 1 CVPR Unit, Indian Statistical Institute, Kolkata- 700108, India \\ roy.anandarup@gmail.com, swapan@isical.ac.in \\ 2 School of Education Tech., Jadavpur University, Kolkata- 700032, India \\ debyaninandi@gmail.com \\ 3 Dept. of Computer \& System Sciences, \\ Visva-Bharati University, Santiniketan- 731235, India \\ roy.utpal@gmail.com
}

\begin{abstract}
This article deals with color image segmentation in the huesaturation-value space. Hue, saturation and value components are samples on a cylinder. A model for such data is provided by the semi-wrapped Gaussian distribution. Further its mixture is used to approximate the hue-saturation-value distribution. The mixture parameters are estimated using the standard EM algorithm. The results are obtained on Berkeley segmentation dataset. Comparisons are made with vM-Gauss mixture model, GMM and Mean-Shift procedures. Experimental results reveal improvement in segmentation by our method.
\end{abstract}

\section{Introduction}

Color image segmentation is becoming important in many applications since color images are now easily available and can provide more information than gray level images. A popular way to represent a color image is the RGB system. However, due to its redundancy, many applications prefer the hue-saturationvalue (HSV) color system. Consequently, segmentation in HSV space becomes crucial. In this article, we apply clustering based segmentation approach. The basic idea is to directly cluster the pixels in a certain color space by employing some clustering algorithms. Such algorithms include Mean-Shift and normalized-cut procedures [1] which are used widely. Segmenting an image into clusters involves determining which clusters generate the image pixels, which is the hidden information. Under this consideration, a mixture model based approach can be used. This approach assumes a mixture distribution approximating the distribution of image pixels. The mixture parameters can be estimated using expectation maximization algorithm. Such an earlier attempt was made by Carson et. al. 2] using Gaussian Mixture Model (GMM). Later, Bougulia et. al. [3] considered the aspect of skewness of color bands and applied a mixture of Dirichlet distributions for the joint distribution of RGB color spectrum.

The HSV system is a mixture of angular (hue) and linear (saturation, value) data. Mere linear mixture models may fail to model the hue distribution. Hue 
can be represented by a random variable $\Theta \in[0,2 \pi)$. A direct modeling of $\Theta$ can be avoided by transforming it into its linear representation $(\cos \theta, \sin \theta)$ and applying a linear mixture model. However the pair $(\cos \theta, \sin \theta)$ may be highly correlated, thus may pose the problem of singularity of the covariance matrix for some mixture components. Instead, we use the wrapped Gaussian distribution 4 for $\Theta$. This distribution is a wrapping of linear Gaussian distribution around a circle. To cope with the circular-linear characteristics we need to wrap only one variable (corresponding to $\Theta$ ) of a multivariate Gaussian distribution, whereas the other unwrapped variables represent linear portion. This distribution is termed as semi-wrapped Gaussian distribution [5]. Its mixture is used to approximate the HSV joint data distribution. An alternative model in hue-saturation (HS) space was suggested by Roy et. al. 6] using a mixture of vM-Gauss distribution. However, joint distribution in HSV space has not been proposed yet. An EM algorithm is designed to estimate the mixture parameters. The Berkeley segmentation Dataset [7] is used to test the performance of the methods. We compare our segmentation with vM-Gauss mixture model, GMM and Mean-Shift algorithms using probabilistic rand index.

\section{Semi-wrapped Gaussian Distributions: Description and Mixture}

Our aim is to devise a mixture of multivariate Gaussian distributions having both circular and linear variables. In this context, the wrapped Gaussian is a suitable choice. Otherwise, the von Mises distribution can replace wrapped Gaussian. However, forming a joint distribution using von Mises is difficult. Thus, in spite of the practical drawbacks in the parameter estimation, the wrapped Gaussian distribution, is chosen. The remaining part of this section presents a brief overview on semi-wrapped Gaussian distribution, and its mixture.

\subsection{Wrapped Gaussian Distribution}

In particular, for $\mathcal{N}_{\mu, \sigma}(x)$ being a univariate Gaussian distribution the wrapped univariate Gaussian distribution is defined as follows.

$$
\mathcal{N}_{\mu^{c}, \sigma^{c}}^{w}(v)=\sum_{w \in \mathbb{Z}} \mathcal{N}_{\mu, \sigma}(v+2 w \pi)
$$

where $v \in[0,2 \pi) . \mathcal{N}_{\mu^{c}, \sigma^{c}}^{w}(v)$ is unimodal and symmetric about $\mu^{c}$. It can be shown that the circular mean and variance $\left(\mu^{c}, \sigma^{c}\right)$ are related to linear mean and variance $(\mu, \sigma)$ by the equations $\mu^{c}=\mu(\bmod 2 \pi)$ and $\sigma^{2}=-2 \log \left(1-\sigma^{c}\right)$. In a similar way the multivariate Gaussian distribution can be wrapped onto a sphere to obtain the wrapped version.

\subsection{Semi-wrapped Gaussian Distribution and Mixture}

Let us consider an HSV image. Then, the hue component is angular, whereas saturation and value components are linear. For such situations, a suitable modeling 
should employ a distribution that is wrapped in the angular and non-wrapped in the linear dimensions. The semi-wrapped Gaussian distribution [5] serves this purpose well enough. For simplicity we assume only the first variable $(\Theta)$ is angular (this really is for our problem) and others (x) linear. With these specifications the semi-wrapped Gaussian distribution can be formed by wrapping the first variable of a multivariate Gaussian distribution. Its density is given by:

$$
\mathcal{N}_{\boldsymbol{\mu}^{c}, \Sigma^{c}}^{s w}(\theta, \mathbf{x})=\sum_{w \in \mathbb{Z}} \mathcal{N}_{\boldsymbol{\mu}, \Sigma}(\theta+2 w \pi, \mathbf{x})
$$

with mean $\boldsymbol{\mu}^{c}$ and covariance matrix $\Sigma^{c}$.

Let us now define the mixture model of $K$ semi-wrapped Gaussian distributions (SWGMM). The density of the mixture is given by:

$$
f(\theta, \mathbf{x} \mid \boldsymbol{\Xi})=\sum_{h=1}^{K} P(h) \mathcal{N}_{\boldsymbol{\Xi}_{h}}^{s w}(\theta, \mathbf{x})
$$

where $P(h)\left(0 \leq P(h) \leq 1\right.$ and $\left.\sum_{h=1}^{K} P(h)=1\right)$ are the mixing proportions, $\mathcal{N} \boldsymbol{\Xi}_{h}^{s w}(\theta, \mathbf{x})$ is the semi-wrapped Gaussian distribution representing the $h^{\text {th }}$ component of the mixture and $\boldsymbol{\Xi}_{h}$ is the set of parameters of the $h^{\text {th }}$ component. The symbol $\boldsymbol{\Xi}=\left(\boldsymbol{\Xi}_{1}, \ldots, \boldsymbol{\Xi}_{K}, P(1), \ldots, P(K)\right)$ refers to the entire set of parameters to be estimated.

\section{Maximum Likelihood Estimation}

Let $\boldsymbol{\aleph}=p_{1}, \ldots, p_{N}$ be a finite set of $N$ samples drawn independently from the SWGMM. Here $p_{i}=\left(\theta_{i}, \mathbf{x}_{i}\right)$ is a circular-linear data. To maximize the likelihood function, the expectation maximization (EM) is widely used. The cluster information and wrapping are the hidden variables. The standard EM settings express the distribution of hidden variables by:

$$
q\left(h, w \mid p_{i}\right)=\frac{P(h) \mathcal{N} \boldsymbol{\mu}_{h}, \Sigma_{h}\left(p_{i}+2 w \pi\right)}{\sum_{l=1}^{K} P(l) \mathcal{N}_{\boldsymbol{\Xi}_{l}}^{s w}\left(p_{i}\right)}
$$

Here $p_{i}+2 w \pi$ indicates the tuple $(\theta+2 w \pi, \mathbf{x})$. The EM algorithm maximizes the $\log$-likelihood modified by the inclusion of $q\left(h, w \mid p_{i}\right)$. To maximize the modified log-likelihood we may go independently for a priory probabilities $P(h)$ and the parameters $\boldsymbol{\Xi}_{h}$. The a priori probabilities can be found out with:

$$
P(h)=\frac{1}{N} \sum_{i=1}^{N} \sum_{w \in \mathbb{Z}} q\left(h, w \mid p_{i}\right) .
$$


The expressions for $\boldsymbol{\mu}_{h}^{c}$ and $\Sigma_{h}^{c}$ are quite similar to the linear Gaussian mixture and obtained using:

$$
\begin{aligned}
\boldsymbol{\mu}_{h}^{c}= & \frac{\sum_{i=1}^{N} \sum_{w \in \mathbb{Z}} q\left(h, w \mid p_{i}\right)\left(p_{i}+2 w \pi\right)}{\sum_{i=1}^{N} \sum_{w \in \mathbb{Z}} q\left(h, w \mid p_{i}\right)} \\
\Sigma_{h}^{c} & =\frac{\sum_{i=1}^{N} \sum_{w \in \mathbb{Z}} q\left(h, w \mid p_{i}\right)\left(p_{i}+2 w \pi-\boldsymbol{\mu}_{h}^{c}\right)\left(p_{i}+2 w \pi-\boldsymbol{\mu}_{h}^{c}\right)^{T}}{\sum_{i=1}^{N} \sum_{w \in \mathbb{Z}} q\left(h, w \mid p_{i}\right)} .
\end{aligned}
$$

In practice $w$ hold values $-1,0$ and 1 . We continue writing $w \in \mathbb{Z}$ for completeness.

\section{Color Image Segmentation}

Let us assume that the hue, saturation and value components of an HSV image arise from a SWGMM (Eq. 3). The EM method estimates mixture parameters by iterating expectation (Eq. 4) and maximization steps (Eqs. 5, 6] and 7). These steps iterate until the log-likelihood stabilizes. We apply K-Means algorithm on HSV image to obtain initial clustering. Initially, $\boldsymbol{\mu}_{h}^{c}$ and $\Sigma_{h}^{c}$ are estimated as follows.

$$
\boldsymbol{\mu}_{h}^{c}=\frac{\sum_{i=1}^{N_{h}} \sum_{w \in \mathbb{Z}}\left(p_{i}+2 w \pi\right)}{N_{h}} \text { and } \Sigma_{h}^{c}=\frac{\sum_{i=1}^{N_{h}} \sum_{w \in \mathbb{Z}}\left(p_{i}+2 w \pi-\boldsymbol{\mu}_{h}^{c}\right)\left(p_{i}+2 w \pi-\boldsymbol{\mu}_{h}^{c}\right)^{T}}{N_{h}} .
$$

The a priori probabilities are set with $P(h)=\frac{N_{h}}{N}$ where $N_{h}$ is the number of elements in $h^{\text {th }}$ cluster. In order to detect the number of clusters automatically we use the Schwarz's Bayesian Information Criterion (BIC). Optimum value of $K$ is the first local minimum of $B I C$. Note, for the gray portions, the hue becomes undefined. We separate out the gray portions before employing EM. A separate clustering should be employed with the gray portions. Yet, here we encounter only a few gray pixels mostly in the background and thus leave them to minimize time complexity.

\section{Evaluation of Segmentation}

To evaluate the quality of segmentation algorithm we have to compare the resulting clusters with the ground-truth segmentations. A good survey on evaluation methodologies can be found in [8]. Recently, Unnikrishnan et. al. 9] proposed a Probabilistic Rand Index (PRI) which is a generalization of classical Rand Index. The PRI is further applied to evaluate a color segmentation procedure designed 

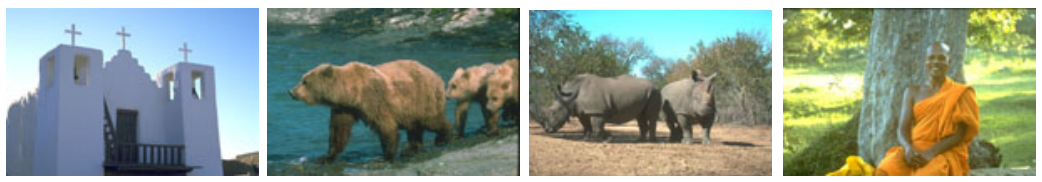

(a)
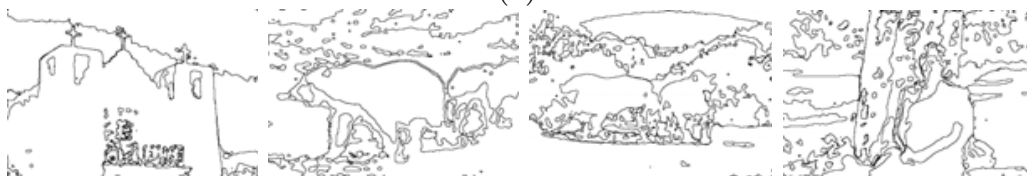

(b)

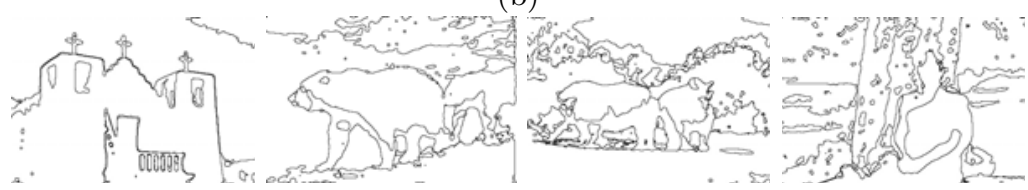

(c)

Fig. 1. (a) Example images of Berkeley segmentation dataset. Image Ids are (from top left to right) "24063", "100075", "112082" and "376020". (b)(c) Corresponding segmentations in HS and HSV space respectively.

by Ilea and Whelan [10. The PRI allows comparison of a test segmentation with multiple ground-truth images by evaluating the pairwise relationships between pixels. It takes values in the range $[0,1]$, where a higher PRI value indicates a better match between the segmented result and the ground-truth data. In this study we use PRI to evaluate the segmentation process.

\section{Results and Discussions}

The Berkeley segmentation dataset [7] contains several color images of natural scenes. The images contain at least one distinguishable and identifiable object embedded in a natural scene. The images we consider have ground-truth in the form of manual segmentation performed by several users independently. Let us now present some results after running the algorithm in HS (Fig. 1(b)) and HSV (Fig. 1(c)) space. Visually it is difficult to assess which segmentation is better. This can be done using the PRI. We compare SWGMM with the mixture model of vM-Gauss distribution (vMGMM), the GMM and the Mean-Shift procedures (last two in RGB). The comparison results are presented in Fig. 2 , The Mean-Shift performs better for a few images. The GMM, on the other hand, outperforms Mean-Shift in most cases. The vMGMM assumes independence of hue and saturation. By noting that SWGMM outperforms vMGMM, we could conclude, the correlation present in hue, saturation and/or value should be crucial for segmentation. The SWGMM in HSV space can show better performance for some image than GMM and Mean-Shift. A detailed statistical study may reveal the significance of the results. We are in a process to design that. 


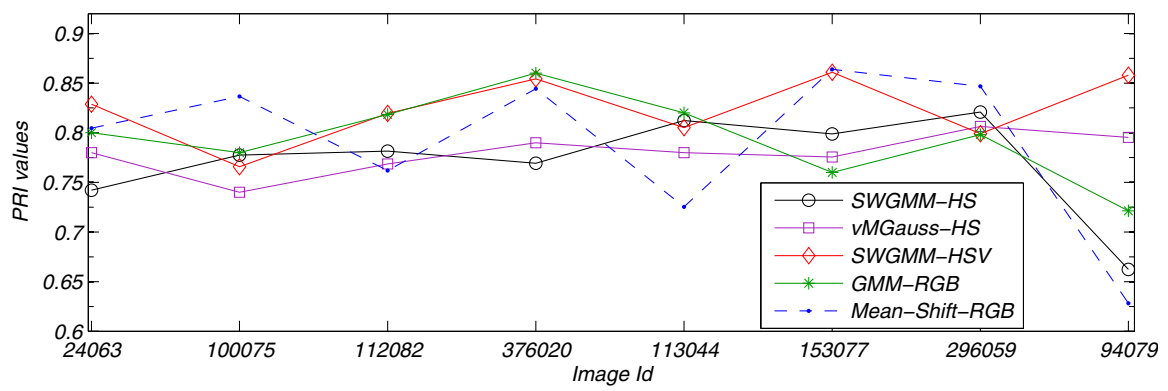

Fig. 2. Comparison of different segmentation algorithms with respect to PRI

\section{Summery and Future Scope}

We study the problem of color image segmentation in HSV space. A mixture of semi-wrapped Gaussian distributions is used to model hue, saturation and value data. Results and comparisons are on Berkeley segmentation dataset. Future work may include the study of different forms of joint distributions on circularlinear data.

\section{References}

1. Tao, W., Jin, H., Zhang, Y.: Color image segmentation based on mean shift and normalized cuts. IEEE Trans. on SMC(B) 37, 1382-1389 (2007)

2. Carson, C., Belongie, S., Greenspan, H., Malik, J.: Blobworld: Image segmentation using expectation-maximization and its application to image querying. IEEE Trans. on PAMI 24, 1026-1038 (2002)

3. Bougulia, N., Ziou, D., Vaillancourt, J.: Unsupervised learning of a finite mixture model based on the dirichlet distribution and its application. IEEE Trans. on Image Processing 13, 1533-1543 (2004)

4. Mardia, K.V., Jupp, P.: Directional Statistics. John Wiley and Sons Ltd., Chichester (2000)

5. Bahlmann, C.: Directional features in online handwriting recognition. Pattern Recognition 39, 115-125 (2006)

6. Roy, A., Parui, S.K., Roy, U.: A color based image segmentation and its application to text segmentation. In: Proc. of Indian Conf. on Computer Vision, Graphics and Image Processing, pp. 313-319 (2008)

7. Martin, D., Fowlkes, C., Tal, D., Malik, J.: A database of human segmented natural images and its application to evaluating segmentation algorithms and measuring ecological statistics. In: Proc. Int Conf. Computer Vision, pp. 416-423 (2001)

8. Chabrier, S., Laurent, H., Rosenburger, C., Marche, P.: A comparative study of supervised evaluation criteria for image segmentation. In: Proc. of the European Signal Processing Conference, pp. 1143-1146 (2004)

9. Unnikrishnan, R., Pantofaru, C., Hebert, M.: Toward objective evaluation of image segmentation algorithms. IEEE Trans. on PAMI 29, 929-944 (2007)

10. Ilea, D.E., Whelan, P.F.: Ctex - an adaptive unsupervised segmentation algorithm based on color-texture coherence. IEEE Trans. on Image Processing 17, 1926-1939 (2008) 